\section{Vivencias de los padres o cuidadores de niños con enfermedad de Hirschsprung o con malformaciones anorrectales, bajo seguimiento después de la cirugía}

\author{
Luis Mauricio Figueroa ${ }^{1}$, Mercy Soto², Jaime Martínez ${ }^{1}$ \\ ${ }^{1}$ Programa de Medicina, Fundación Universitaria Autónoma de las Américas, Pereira, Colombia \\ ${ }^{2}$ Programa de Enfermería, Facultad de Ciencias de la Salud, Universidad Libre, Pereira, \\ Colombia
}

Introducción. La enfermedad de Hirschsprung y las malformaciones anorrectales son enfermedades que afectan a los niños y comprometen su vida desde el nacimiento, por lo que su diagnóstico y tratamiento temprano son esenciales. Las comorbilidades que implican a largo plazo llevan a los padres y cuidadores a situaciones que pueden generar graves complicaciones para el niño si no se cuenta con el acompañamiento adecuado. Objetivo. Describir y analizar las vivencias de los padres de niños con enfermedad de Hirschsprung o malformaciones anorrectales bajo seguimiento tras haber sido sometidos a cirugía.

Materiales y métodos. Se hizo un estudio cualitativo con un enfoque fenomenológico mediante entrevistas a cinco padres de niños con enfermedad de Hirschsprung y a otros cinco de niños con malformaciones anorrectales.

Resultados. Se encontraron experiencias comunes, de las cuales surgieron los siguientes temas: a) el diagnóstico difícil, debido a los sentimientos y al impacto generados por la noticia, situación que no siempre es detectada tempranamente por el personal de salud; b) el tratamiento, el cual provoca aislamiento social por las hospitalizaciones y la presencia de una ostomía, además de que los recursos se concentran en el niño en detrimento del hogar; se requiere capacidad de afrontamiento para lograr la reparación quirúrgica definitiva, y c) el contexto, ya que las instituciones de salud pueden convertirse en barreras; además, el personal de salud debe disponer de mayor educación al respecto y son necesarias las redes de apoyo social.

Conclusión. Las vivencias reflejan que el diagnóstico, el tratamiento y el contexto generan un gran impacto en las vidas de los padres o cuidadores de niños con estas condiciones.

Palabras clave: malformaciones anorrectales/diagnóstico; enfermedad de Hirschsprung/ diagnóstico; cuidadores; cirugía.

Recibido: 28/09/17

Aceptado: 09/08/18

Publicado: 09/08/18

Citación:

Figueroa L, Soto M, Martínez J. Vivencias de los padres o cuidadores de niños con enfermedad de Hirschsprung o con malformaciones anorrectales, bajo seguimiento después de la cirugía. Biomédica. 2018;39:147-56

https://doi.org/10.7705/biomedica.v39i1.3927

\section{Correspondencia:}

Luis Mauricio Figueroa, Villa Sol MB, Carrera 19 No 94-96, C. 19 , Pereira, Colombia

Teléfonos: (576) 3213465 y (313) 7371166

luis.figueroa@uam.edu.co

Contribución de los autores:

Luis Mauricio Figueroa y Mercy Soto: concepción y diseño del estudio, recolección y análisis de la información y redacción del manuscrito

Jaime Martínez: adquisición, análisis e interpretación de los datos

Financiación:

La investigación fue financiada por la Fundación Universitaria Autónoma de las Américas y por la Universidad Libre, seccional Pereira.

\section{Conflicto de intereses:}

Los autores declaramos no tener conflicto de intereses.
Experiences of parents and/or caretakers of children with Hirschsprung's disease or anorectal malformations during follow-up after pediatric surgery

Introduction: Hirschsprung's disease and anorectal malformations are pathologies that affect the pediatric population and compromise life from birth, making diagnosis and early treatment essential. The comorbidities they lead to in the long-term take parents and caretakers to situations that, without proper accompaniment, can generate serious complications in the child.

Objective: To describe the meaning of the experiences of parents of children with Hirschsprung's disease or anorectal malformations who have had surgical treatment and are being followed-up.

Materials and methods: This was a phenomenological qualitative research carried out through interviews with five parents of children with Hirschsprung's disease and five parents of children with anorectal malformations.

Results: We found common experiences from which the following topics emerged: a) Difficult diagnosis, which involves the feelings and the impact generated by receiving this news; for health personnel it is not always a situation that is identified early; b) treatment: Social isolation due to hospitalizations and the presence of an ostomy, in addition to the channeling of resources for the child at the expense of those of the home; coping skills are required to achieve definitive surgical repair; and c) context: Health institutions may become barriers and health personnel must have more education in this regard; social support networks are necessary.

Conclusion: The experiences reflected that diagnosis, treatment, and context generated great impact on the lives of parents and/or caretakers of children with these pathologies.

Key words: Anorectal malformations/diagnosis; Hirschsprung disease/diagnosis; caregivers; surgery. 
La enfermedad de Hirschsprung es una alteración del desarrollo del sistema nervioso entérico, que se caracteriza por la ausencia de células ganglionares en una longitud variable del intestino, lo que ocasiona una obstrucción funcional (1). Después del nacimiento, los infantes afectados presentan tempranamente signos y síntomas de obstrucción intestinal $(2,3)$; su incidencia es de uno en 5.000 nacidos vivos, siendo la aganglionosis confinada al intestino rectosigmoides, conocida como de segmento corto, la más frecuente $(2,3)$.

Más de las dos terceras partes de los pacientes con esta condición se diagnostican durante el periodo neonatal y casi el $95 \%$ antes del año de edad $(4,5)$. En el grupo restante los episodios que se manifiestan, como retardo en la expulsión de meconio, distensión abdominal y poca tolerancia a la alimentación, suelen ser erróneamente interpretados por el personal de salud como estreñimiento, en tanto que los eventos de infecciones gastrointestinales recurrentes y vómito por enterocolitis no se detectan oportunamente (6), lo cual expone a los pacientes a morbilidad preoperatoria y posoperatoria (7).

En los casos de diagnóstico tardío, la mayoría de las veces se impone un tratamiento por etapas, lo que somete al paciente a una colostomía y a su posterior cierre, situaciones que conllevan aumento en la morbilidad y la mortalidad (8) y, además, alteraciones del comportamiento y del entorno social, familiar y económico, entre otros, que al final deterioran la calidad de vida del paciente y la del grupo familiar (9). Por ello, es ideal practicar la reparación tan temprano como sea posible, de la manera menos invasiva y en una sola intervención (1).

Otras enfermedades colorrectales frecuentes que afectan a los neonatos, son las malformaciones anorrectales, grupo de anomalías que representa un componente importante de la práctica quirúrgica pediátrica, con una incidencia de 1:1.500 a 1:5.000 nacidos vivos y una presentación clínica variable, desde formas menos complejas a situaciones que se convierten en un reto que requiere cirugías en varias etapas (1). Dado su amplio espectro, existen diferentes formas de presentación en los niños, las cuales pueden afectar, además del ano y el recto, las vías genitales y urinarias, y determinan que unos pacientes tengan un excelente pronóstico y otros presenten un serio compromiso de la funcionalidad con pobres resultados a largo plazo (1).

A pesar del examen físico de rutina después del parto, en la literatura médica se han llegado a reportar elevados porcentajes, entre el 26 y el $57,9 \%$, de casos de diagnóstico tardío de estas malformaciones $(10,11)$. El retardo se define como el diagnóstico después de 24 horas del nacimiento 0 la remisión a un centro de referencia de tercer nivel después de más de 24 horas del diagnóstico, con lo cual se incrementan la morbilidad y la mortalidad (11); la mayoría de estos pacientes presenta síntomas obstructivos (86 \%), de distensión abdominal (57\%) y retardo en el paso del meconio ( $29 \%$ ) (12). El retraso en el diagnóstico puede llevar a complicaciones evitables del orden del $19 \%$ e, incluso, una mortalidad de hasta el $4 \%$ (13), por lo que son esenciales la práctica de un buen examen perineal y la educación del personal de salud para evitar estas situaciones $(11,14)$.

Partiendo de las experiencias de los pacientes y de sus padres o cuidadores, durante las últimas dos décadas han surgido en diferentes partes del mundo instituciones orientadas a brindar apoyo, información y educación a los pacientes y a su grupo familiar, lo cual ha permitido el desarrollo de diversas estrategias de colaboración entre padres y pacientes, de un lado, y el personal de salud, del otro, con probados beneficios para todos en los campos de acción común (15-19). 
El objetivo de este trabajo fue describir el significado de las vivencias de los padres o cuidadores de niños con enfermedad de Hirschsprung o malformaciones anorrectales, bajo seguimiento por haber sido sometidos a tratamientos quirúrgicos.

\section{Materiales y métodos}

Se hizo un estudio cualitativo con enfoque fenomenológico e interpretativo, siguiendo la perspectiva propuesta por Merleau Ponti (20), para develar mediante entrevistas a profundidad con los padres de niños afectados por la enfermedad de Hirschsprung o por malformaciones anorrectales, sus vivencias en cuanto al diagnóstico, el tratamiento y el contexto familiar y social.

Se tuvieron en cuenta los criterios éticos y bioéticos contenidos en la Declaración de Helsinki de 1964 para investigaciones con seres humanos y en la Resolución 8430 de 1993 adoptada por el Ministerio de Salud, y se obtuvo el aval del Comité de Bioética de la Fundación Universitaria Autónoma de las Américas.

Se aplicó un muestreo intencional y se invitó a participar en el estudio a padres o cuidadores de niños con enfermedad de Hirschsprung o malformaciones anorrectales, bajo seguimiento después de la cirugía, que cumplían con los criterios de inclusión: ser mayor de edad, convivir en forma permanente con el niño, poseer las condiciones físicas y mentales para responder a las entrevistas, aceptar la participación voluntaria en el estudio y firmar el consentimiento informado.

Los participantes se seleccionaron de las bases de datos de un servicio de cirugía pediátrica y se acordaron visitas domiciliarias para hacer las entrevistas a profundidad hasta captar el fenómeno, es decir, la experiencia de los aspectos significativos, y descubrir un patrón predecible y consistente a partir de la información aportada por todos los participantes, que se evidenciara eventualmente en unidades de significado para cada tema (21). Se logró la participación de diez padres de familia, cinco para cada enfermedad.

Las entrevistas a profundidad se hicieron mediante preguntas centradas en las vivencias de los participantes como padres o cuidadores de niños con estas condiciones. Las entrevistas se llevaron a cabo en espacios privados y, tras un acercamiento para introducir la conversación, se les solicitó que contaran su experiencia como padres o cuidadores del niño en los momentos del diagnóstico y el tratamiento, así como para sobrellevar la enfermedad de sus hijos en el contexto social. Los diferentes aspectos se fueron entrelazando en un diálogo tranquilo y abierto en el cual los participantes pudieron expresar libremente sus vivencias. Para salvaguardar la identidad de los participantes, se les asignó un código (iniciales de los nombres y números) en secuencia, y las entrevistas se transcribieron posteriormente. Mediante un proceso de lectura y relectura, se descubrieron las estructuras latentes de los contenidos para captar el significado de las experiencias e integrar los hallazgos reveladores de aspectos que trascendían la apariencia de las cosas (22).

Según lo propuesto por Castillo, et al. (21), se aplicaron los siguientes criterios de rigor: credibilidad, en la medida en que los participantes reconocieron como verdaderos los hallazgos reportados por los investigadores; auditoría, ya que se elaboró un registro con la documentación completa de las 
grabaciones, las transcripciones, las notas de campo y los análisis realizados, y transferibilidad o aplicabilidad, es decir, la posibilidad de aplicar los resultados a otras poblaciones en contextos similares, para lo cual se describieron detalladamente los hallazgos que sirven de respaldo a cada tema.

\section{Resultados}

Las entrevistas giraron en torno a los momentos vividos relacionados con el diagnóstico, el tratamiento, y el contexto familiar y social.

Diagnóstico: "Momento muy difícil e inesperado". Al referirse al diagnóstico, los participantes manifestaron haber vivido esta situación como un "momento muy difícil" por enfrentarse a una enfermedad desconocida, no solo por ellos, sino por el personal de salud, pues percibieron las dificultades para hacer un diagnóstico oportuno.

Debido al desconocimiento de la enfermedad de Hirschsprung y de las malformaciones anorrectales, les fue difícil aceptar el diagnóstico, ya que no habían escuchado antes los términos en los que se les habló y, además, fue inesperado saber que sus niños tenían una enfermedad o malformación que afectaba sus vidas.

"Eso fue un momento muy difícil porque no sabía qué era eso, nunca había escuchado esa palabra: ano imperforado. Es más, yo no conocí a nadie que tuviera lo mismo que mi bebé; yo los vine a conocer fue acá, cuando mi bebé estaba hospitalizada".

"La niña tenía 15 días de nacida y fuimos nuevamente a consulta porque ella no había defecado y el neonatólogo me dijo: 'Sí, ella tiene un megacolon o síndrome de Hirschsprung, pero tranquila, le hacemos una colostomía y cuando tenga 10 meses la operamos'... Yo lo miraba asombrada, desconcertada... y yo decía, es que no estoy comprendiendo, no estoy asimilando esto, y mi hermana me miraba y me decía ¿este señor qué es lo que está diciendo?".

Además, los padres contaron que, en los exámenes realizados durante el embarazo, no se había detectado ninguna alteración que los alertara sobre la condición de su bebé, lo que aumentó el desconcierto.

"Porque uno siempre se pregunta: ¿y por qué nació así?"

"Pero yo siempre decía ¿por qué me había tocado a mí?, ¿qué habíamos hecho mal? porque a tantas personas le nacen bien y ¿por qué me tocó a mí? Eso fue inesperado".

Asimismo, los padres consideraron que establecer el diagnóstico fue difícil para el personal de salud, y que no fue oportuno, ya que en ninguno de los diez casos se hizo en el momento del nacimiento y algunos bebés fueron enviados a casa sin que hubieran expulsado el meconio. Además, los participantes refirieron que hubo falencias en el momento de comunicarles el diagnóstico, pues el lenguaje técnico empleado no facilitó que comprendieran y asimilaran la situación que tenían que afrontar.

"La bebé no hizo meconio, yo salí de la clínica, me dijeron que si no defecaba, regresara para estimularla; al día siguiente al consultar la manipularon y lograron que pasara meconio, pero seguía sin hacer espontáneamente. Después de múltiples consultas, contestaron: vamos 
entonces a hacerle una radiografía para descartarle un megacolon y ya luego con el pediatra se dieron cuenta del diagnóstico".

"Bueno en el momento no nos dimos cuenta, porque nadie lo miró, nadie tomó esa precaución, inclusive, lo revisaron y me dijeron que el bebé estaba muy bien, pero no se tomó la precaución de mirarle la colita y darse cuenta que no tenía ano".

Tratamiento: "entre el dolor, la lucha, la tristeza y la esperanza".

Inmediatamente después de que los padres recibieron la noticia del diagnóstico, se les informó que sus bebés debían ser sometidos a un procedimiento quirúrgico de urgencia que en la mayoría de los casos implica una colostomía, lo cual generó en ellos sentimientos de dolor. Además, para el cuidado de sus hijos requerían insumos médicos que el sistema de salud no autoriza con facilidad, lo que provocó su enojo y angustia por la lucha constante para conseguir el tratamiento necesario para los niños. Asimismo, durante la evolución de la enfermedad, experimentaron tristeza por las recaídas a causa de las enterocolitis, que ocasionaron múltiples hospitalizaciones.

Los participantes enfrentaron con angustia las experiencias vividas durante las etapas del diagnóstico y el tratamiento, lo cual se acentuó en el momento del egreso, dada la ausencia de profesionales que les brindaran educación sobre los cuidados que debían prestarle a sus niños en el hogar. Por ello, regresaron a casa llenos de temores sobre el seguimiento, la dieta, la colostomía, el manejo de la herida y las dilataciones anales, aspectos que requerían atención por parte de los profesionales y las instituciones.

"Fue muy doloroso cuando me dijeron que le tenían que hacer una colostomía, él apenas empezando a vivir y ya con una cirugía... Yo me asusté mucho porque ver la colostomía era como verle los intestinos".

Enfrentarse a la colostomía de sus bebés fue algo doloroso, ya que pensaban que la exposición de los intestinos exigía limpiarlos y eso podía causarles daño. Además, los niños y sus madres experimentaron aislamiento social, pues el olor y la necesidad de limpieza frecuente hacía incómodo estar fuera de la casa.

"Siempre fue incómodo... había que cambiarle la colostomía... y así, cómo salíamos a la calle".

"Uno se va aislando y se va olvidando de uno, yo siento que todo pasa a segundo plano porque tengo que cuidarlo".

Los padres refirieron que sus hijos afectados por estas condiciones requirieron incontables hospitalizaciones, tanto por las intervenciones quirúrgicas como por las complicaciones derivadas del tratamiento y de la enfermedad.

"Las hospitalizaciones han sido incontables, por las crisis que le daban..., los médicos decían que tenía enterocolitis".

"Como ella tenía un solo orificio..., es decir, una cloaca, le daban muchas infecciones en la orina... Una vez estuvimos dos meses hospitalizados".

Para los padres estas enfermedades generaron un alto costo económico, ya que debieron asumir los gastos de muchos insumos necesarios que no los cubre el sistema de salud; asimismo, en ocasiones, el manejo interdisciplinario 
requerido no estaba disponible en las instituciones y les correspondió a las familias cubrir los costos adicionales de las consultas con especialistas, así como los desplazamientos a otras ciudades en algunos casos.

"Tuvimos problemas para que nos dieran las barreras y las bolsas para la colostomía, incluso nos tocaba comprarlas y eran cosas muy costosas".

"Mi niña nació con una cloaca, y necesitaba citas con el nefrólogo porque además tenía reflujo del riñón derecho y las citas eran en otra ciudad, varias veces tuvimos que asumir el costo porque la clínica no contaba con uno y la niña necesitaba urgente la consulta".

Según los padres, las instituciones de salud se convirtieron en una barrera para la atención prioritaria que requerían sus hijos. Percibían que las autorizaciones de procedimientos, hospitalizaciones y dotación de los insumos necesarios para cumplir con el tratamiento, no eran oportunas y, en ocasiones, tuvieron que recurrir a mecanismos jurídicos para garantizar la prestación del servicio, lo que consideraron como una 'lucha' por el bienestar de su hijo.

"Se demoraban mucho para autorizar las cirugías".

"No me autorizaban la entrega de los dilatadores, no sabían qué era eso. Todo es demorado para que lo autoricen".

"Recurrimos a recursos jurídicos que obligaran a que las necesidades de atención e insumos fueran cubiertas".

A pesar de su incertidumbre, los padres eran conscientes de la necesidad de afrontar la situación y lograr la recuperación de sus hijos, aferrándose a los profesionales que los atendían con empatía, como los cirujanos, quienes les explicaron en qué consistía el diagnóstico, cuáles eran los retos del tratamiento, los cuidados requeridos, los compromisos que debían asumir frente al seguimiento y, además, trabajaron de manera coordinada con un equipo interdisciplinario que acompañó a las familias. Por ello, los padres los consideraban personas dignas de confianza que les permitían mantener la esperanza.

"Él era muy claro... todo lo explicaba, nos decía qué iba a pasar y eso nos daba confianza".

Por último, los padres desarrollaron la capacidad de afrontamiento que les permitió concentrar sus esfuerzos para lograr el objetivo de la recuperación, representada para ellos en la reparación quirúrgica definitiva y la garantía de una vida lo más funcional posible para sus hijos.

"Tenemos que seguir adelante para encontrar la luz al final del túnel".

"Cuando le hicieron el ano y le cerraron la colostomía, ya se siente uno feliz".

Contexto: "se requiere de apoyo social para compartir las experiencias".

El apoyo social es un medio importante para brindar y recibir ayuda, y permite el intercambio de conocimientos a partir de la experiencia vivida por otros padres que enfrentan una situación similar, por lo que los participantes consideraron que esta es una forma de motivación y apoyo para superar las angustias propias del diagnóstico y del tratamiento. 
"Es aterrador, pensar que lo vamos a traer para la casa; bueno, cuando uno está en la clínica, está con médicos y enfermeras, lo que más me aterraba era el manejo en la casa".

"Fue horrible hacerle las dilataciones, eso me pareció estresante, pensar que podría hacerle daño. Pero el esposo mío siempre estuvo ahí conmigo diciéndome, el bebé nos necesita porque él va a depender de nosotros dos. Él siempre estaba allí conmigo y eso me ayudó mucho para seguir adelante".

La familia, como primera línea de apoyo social, es el medio más cercano $y$, por ello, es necesario educar a sus integrantes en el cuidado de la enfermedad para fortalecer el vínculo familiar que facilite el intercambio de las ayudas requeridas para el bienestar del niño.

"Es importante contar las experiencias vividas, que brinden orientación... y compartirlas para contarles que mi niño tuvo una colostomía y explicarles cómo es el cuidado... Uno tiene que darle apoyo a la gente para que lo enfrenten, así como lo hemos enfrentado nosotros; que no es fácil, pero se puede lograr la recuperación del niño".

Los padres percibieron que ayudar a otros en situación similar era una forma de agradecer la recuperación de sus hijos y de dar ejemplo y motivación, por lo cual consideraron necesarias las redes de apoyo social.

\section{Discusión}

Los padres de niños con enfermedad de Hirschsprung o con malformaciones anorrectales, comparten situaciones y experiencias de las cuales surgen temas comunes que permiten describir el fenómeno vivido y que estuvieron presentes en los participantes del estudio.

Tal como lo describen Peña, et al. (1), los padres mencionaron su felicidad y las grandes expectativas y esperanzas de tener un niño sano, por lo que recibir la noticia de que sufría una de estas condiciones en términos completamente desconocidos, los sumía en un estado de incertidumbre y desconsuelo, acrecentado por la información de que su bebé debía ser sometido a una intervención quirúrgica con diferentes posibilidades de morbilidad y mortalidad.

El diagnóstico de estas condiciones puede causar miedo, estrés y preocupación en los padres (23), principalmente, porque se trata de enfermedades raras y desconocidas para ellos, que afectan órganos y áreas anatómicas consideradas cercanas al tabú (24). En el estudio de Funakosi, et al., sobre la ansiedad de padres cuyos hijos presentaban estas enfermedades, se estableció que las razones de la ansiedad se debían a la carga psicológica de dar a luz niños con discapacidad, lo cual se agravó ante la necesidad de someterlos a una cirugía y las preocupaciones sobre los cuidados posoperatorios, los cuales incluyen dilataciones e irrigaciones rectales, entre otros, que pueden afectar el estado mental de los padres o cuidadores $(25,26)$. Estos hallazgos coinciden con el tema "momento muy difícil e inesperado" determinado en el presente estudio.

Con respecto al diagnóstico, el personal de salud involucrado en el cuidado neonatal de los niños con estas condiciones debe desarrollar un acusado sentido de la sospecha para llevar a cabo un diagnóstico precoz, ya que las decisiones terapéuticas que se tomen durante las primeras horas de vida serán cruciales para el futuro del niño y los enfoques inadecuados 
pueden tener repercusiones en la calidad de vida del paciente (1). En el presente estudio, los padres percibieron que el diagnóstico fue tardío, retardo que les ocasionó mayor incertidumbre y expuso a sus hijos a procedimientos adicionales y a la resolución de la anomalía por etapas, lo que es evitable con la detección oportuna $(6,7)$.

En cuanto al tratamiento, la decisión de hacer una colostomía a una edad tan temprana, sumada a los múltiples procedimientos diagnósticos y quirúrgicos, los cuidados posoperatorios, como las dilataciones, las barreras institucionales y el alto costo asumido por los padres y cuidadores, generaron en los participantes sentimientos negativos, lo que concuerda con los hallazgos de Muzira, et al., quienes detectaron, en los padres de niños con una situación similar, emociones de culpa, vergüenza, tristeza, aislamiento, estigma, pérdida de la dignidad y dolor, entre otros. Además, se estableció que estas enfermedades comprometían la estabilidad familiar y económica, y restaban oportunidades de vincularse a la vida laboral por la demanda de cuidados del niño (27).

En cuanto a las necesidades de educación, apoyo social y confianza en el grupo interdisciplinario tratante, siempre debe tenerse en cuenta que el nacimiento de un niño con una anomalía no es solamente un reto quirúrgico, sino también una situación que afecta intensamente a los padres y la familia. Ningún padre que no sea médico puede imaginar cómo estas enfermedades, los esfuerzos que implica la solución definitiva y la funcionalidad futura, afectarán el desarrollo de su hijo (15). No hace mucho más de seis décadas, el diagnóstico de una de estas condiciones era una sentencia de muerte, pero como los tratamientos son cada vez más exitosos, otros aspectos esenciales como la calidad de vida y la evolución futura cobran cada vez más importancia. Son el paciente y su familia, no el cirujano, quienes cargan con el cuidado cotidiano después del procedimiento quirúrgico y viven con las consecuencias a largo plazo (1).

En las últimas dos décadas, se han organizado en diferentes partes del mundo instituciones de apoyo a las personas y a los grupos familiares afectados por esta situación, con el fin de brindar soporte, información y educación. Ello ha permitido el desarrollo de diversas estrategias de colaboración entre padres y pacientes, de un lado, y el personal de salud, del otro (15-18). Estas organizaciones de apoyo ilustran la importancia particular de los grupos de autoayuda para enfermedades raras, lo que entraña beneficios para las personas afectadas y sus familiares, pero también para el personal encargado de su atención. Los efectos sinérgicos de estas redes integradoras se han fortalecido aún más por la globalización y el internet, aunque aún hoy los campos de acción y las ventajas del apoyo mutuo con frecuencia se subestiman (19).

A pesar de las diferencias en el diagnóstico, los miembros de estas redes de apoyo encuentran puntos comunes en torno a las condiciones que aquejan a sus hijos, los procedimientos terapéuticos, las consecuencias a largo plazo y el efecto en su calidad de vida, aspectos sobre los cuales requieren información, educación, respaldo, y consejo para ellos y para sus familias (16). Esto convierte a estos grupos de apoyo en instituciones que ofrecen servicios invaluables, incluso para el personal médico y paramédico. Entre las estrategias de educación, se programan reuniones, conferencias y actividades lúdicas que permiten compartir y aprender lecciones de las experiencias de unos y otros (28). 
En resumen, el desarrollo de redes de apoyo de padres tiene beneficios evidentes para los pacientes y sus familias, pero también para los prestadores de servicios de salud. Cuando los padres se encuentran con otros que han sufrido sus mismos padecimientos, se dan cuenta de que no están solos ni son los únicos en el mundo y que, por el contrario, son muchos los individuos afectados de formas menos o más graves que ellos mismos, pero, además, se percatan de los éxitos, dificultades, complicaciones y resultados de los equipos de salud y, de esta manera, van venciendo sus miedos y adquiriendo mayor confianza en los grupos médicos especializados y en sí mismos.

\section{Agradecimientos}

A la Fundación TRAER Vida y Futuro.

\section{Referencias}

1. Peña A, Bischoff A. Surgical treatment of colorectal problems in children. First edition. Heidelberg: Springer; 2013. p. 480.

2. Wester T, Granström AL. Hirschsprung disease - Bowel function beyond childhood. Semin Pediatr Surg. 2017;26:322-7. https://doi.org/10.1053/j.sempedsurg.2017.09.008

3. Levitt MA, Dickie B, Peña A. The Hirschsprung patient who is soiling after what was considered a "successful" pull-through. Semin Pediatr Surg. 2012;21:344-53. https://doi. org/10.1053/j.sempedsurg.2012.07.009

4. Kapur RP, Kennedy AJ. Transitional zone pull through: Surgical pathology considerations. Semin Pediatr Surg. 2012;21:291-301.https://doi.org/10.1053/i.sempedsurg.2012.07.003

5. López JC, Jaramillo LE. Utilidad de la biopsia rectal en el estudio del paciente pediátrico con estreñimiento. Experiencia de cinco años en un centro colombiano de referencia. Rev Fac Med. 2011;59:237-43.

6. Maerzheuser S, Bassir C, Rothe K. Hirschsprung disease in the older child: Diagnostic strategies. Clin Pediatr. 2012;51:1087-90. https://doi.org/10.1177/0009922812458354

7. Lee CC, Lien R, Chian MC, Yang PH, Chu SM, Fu JH, et al. Clinical impacts of delayed diagnosis of Hirschsrpung's disease in newborn infants. Pediatr Neonatol. 2012;53:133-7. https://doi.org/10.1016/j.pedneo.2012.01.011

8. Ekenze SO, Agugua-Oblanyo NE, Amah C. Colostomy for large bowel anomalies in children: A case controlled study. Int J Surg. 2007;5:273-7. https://doi.org/10.1016/j. ijsu.2007.01.008

9. Guerrero S, Angelo M. Impacto del estoma enteral en el niño y su familia. Av Enferm. 2010;28:99-108. https://doi.org/10.15446/av.enferm

10. Theron A, Numanoglu A. Birth prevalence of anorectal malformations for the Western Cape Province, South Africa, 2005 to 2012. Eur J Pediatr Surg. 2017;27:449-54. https://doi. org/10.1055/s-0036-1597945

11. Govender S, Wiersma R. Delayed diagnosis of anorectal malformations (ARM): Causes and consequences in a resource-constrained environment. Pediatr Surg Int. 2016;32:36975. https://doi.org/10.1007/s00383-016-3866-5

12. Wilson B, Etheridge C, Soundappan S, Holland A. Delayed diagnosis of anorectal malformations: Are current guidelines sufficient? J Paediatr Child Health. 2010;46:268-72. https://doi.org/10.1111/j.1440-1754.2009.01683.X

13. Haider N, Fisher R. Mortality and morbidity associated with late diagnosis of anorectal malformations in children. Surgeon. 2007;5:327-30.

14. Turowski C, Dingemann J, Gillick J. Delayed diagnosis of imperforate anus: An unacceptable morbidity. Pediatr Surg Int. 2010;26:1083-6. https://doi.org/10.1007/s00383010-2691-5

15. Holschneider AM, Hutson JM. Anorectal malformations in children. First edition. Heidelberg: Springer-Verlag; 2006. p. 3.

16. Pull-thru Network. Glossary. Normal, United States. Fecha de consulta: 10 de septiembre de 2016. Disponible en: http://www.pullthrunetwork.org/Pages/Detailld/48 
17. SoMA EV. Syndrome Fehlbildungs-Assoziationen und -Komplexe in Kombination mit anorektalen Malformationen und mit Morbus Hirschsprung. Munich, Alemania. Fecha de consulta: 10 de septiembre de 2016. Disponible en: http://soma-ev.de/fileadmin/Dokumente/ downloads/Allgemein/Medizinische Infos/2017 SoMA Broschuere Syndrome finalWeb.pd

18. National Organization for Rare Disorders. International joint recommendations to address specific needs of undiagnosed rare disease patients. The Rare Disease Database. Danbury, United States. Fecha de consulta: 10 de septiembre de 2016. Disponible en: http:// download2.eurordis.org.s3.amazonaws.com/documents/pdf/Undiagnosed-InternationalJoint-Recommendations.pdf

19. Jenetzky E, Schwarzer N. Not alone with a rare disease. The importance of self-help given by the example of anorectal malformations. Bundesgesundheitsblatt Gesundheitsforschung Gesundheitsschutz. 2008;51:529-38. https://doi.org/10.1007/s00103-008-0525-6

20. Merleau Ponti M. Phenomenology of perception. New York, Routledge, 1962. p. 567.

21. Castillo E, Vásquez M. El rigor metodológico en la investigación cualitativa. Colomb Med. 2003;34:164-7.

22. Ayala R. Formación de investigadores de las ciencias sociales y humanas en el enfoque fenomenológico hermenéutico (de van Manen) en el contexto hispanoamericano. Educación XX1. 2016;19:359-81. https://doi.org/10.5944/educXX1.13945

23. Witvliet M, Sleeboom C, de Jong J, van Dijk A, Zwaveling S, van der Steeg A. Anxiety and quality of life of parents with children diagnosed with an anorectal malformation or Hirschsprung disease. Eur J Pediatr Surg. 2014;24:70-4. https://doi. org/10.1055/s-0033-1353491

24. Schwarzer $\mathbf{N}$. How could self-help support patients and families with anorectal malformations? -Psychosocial help offers of SoMA eV. Prax Kinderpsychol Kinderpsychiatr. 2010;59:5-21. https://doi.org/10.13109/prkk.2010.59.1.5

25. Funakosi S, Hayashi J, Kamiyama T, Ueno T, Ishii T, Wada M, et al. Liaison-consultation for the children who have undergone repair of imperforate anus and Hirschsprung disease. $J$ Pediatr Surg. 2005;40:1156-62. https://doi.org/10.1016/j.jpedsurg.2005.03.059

26. Streisand R, Braniecki S, Tercyak KP, Kazak AE. Childhood illness related parenting stress: The pediatric inventory for parents. J Pediatr Psychol. 2001;26:155-62.

27. Muzira A, Kakembo N, Kisa P, Langer M, Sekabira J, Ozgediz D, et al. The socioeconomic impact of a pediatric ostomy in Uganda: A pilot study. Pediatr Surg Int. 2018;34:457-466. https://doi.org/10.1007/s00383-018-4230-8

28. Gribbin E, Croaker D. Long-term follow-up of paediatric anorectal anomalies and the role and efficacy of parent support groups for such conditions. Pediatr Surg Int. 2013;29:931-6. https://doi.org/10.1007/s00383-013-3360-2 